\title{
Localised atypical erythema toxicum neonatorum in a term infant
}

\author{
Pranav Gupta $^{1}$, Payal Mittal ${ }^{1}$, Vandana Sharma ${ }^{2}$, Veena Devgan ${ }^{1}$
}

Sri Lanka Journal of Child Health, 2014; 43(4): 237-238

(Key words: Erythema toxicum neonatorum; atypical)

\section{Introduction}

Erythema toxicum neonatorum (ETN) is a common pustular eruption occurring in $40-70 \%$ of neonates ${ }^{1}$. It is more common in term babies weighing more than $2500 \mathrm{~g}$ with no predilection for race or $\operatorname{sex}^{2,3,4}$. Its incidence decreases proportionally with decreasing maturity and birth weight ${ }^{5}$. ETN commonly appears on the second or third day of life and resolves spontaneously over 5 to 7 days, leaving no sequelae ${ }^{2}$.

Aetiology of ETN is not known, though several hypotheses have been suggested ${ }^{2}$. It may be a transient manifestation of an atopic diathesis ${ }^{4}$, an immediate hypersensitivity reaction ${ }^{6}$, mechanical or thermal stimulation ${ }^{4}$, obstruction of the pilosebaceous duct $^{7}$, or a form of graft-versus-host reaction induced by transplacentally transferred maternal lymphocytes ${ }^{8}$.
The diagnosis of ETN is primarily based on clinical appearance. In this report we describe an atypical case of ETN with localised eruption.

\section{Case report}

The 40 week healthy male infant with a birth weight of $2900 \mathrm{~g}$ was born by normal vaginal delivery and had no skin eruptions at birth. Skin eruptions, according to the mother, had appeared abruptly at around 12 hours of life. The infant had numerous firm and discrete pustules 1 to $2 \mathrm{~mm}$ in diameter, localised to bilateral upper eye-lids and pinna (Figure.1) with normal background skin with only mild erythema. No rash was observed on the trunk, palms or soles. The umbilical stump and the periumbilical area were free from infections. Mucous membranes were not involved.

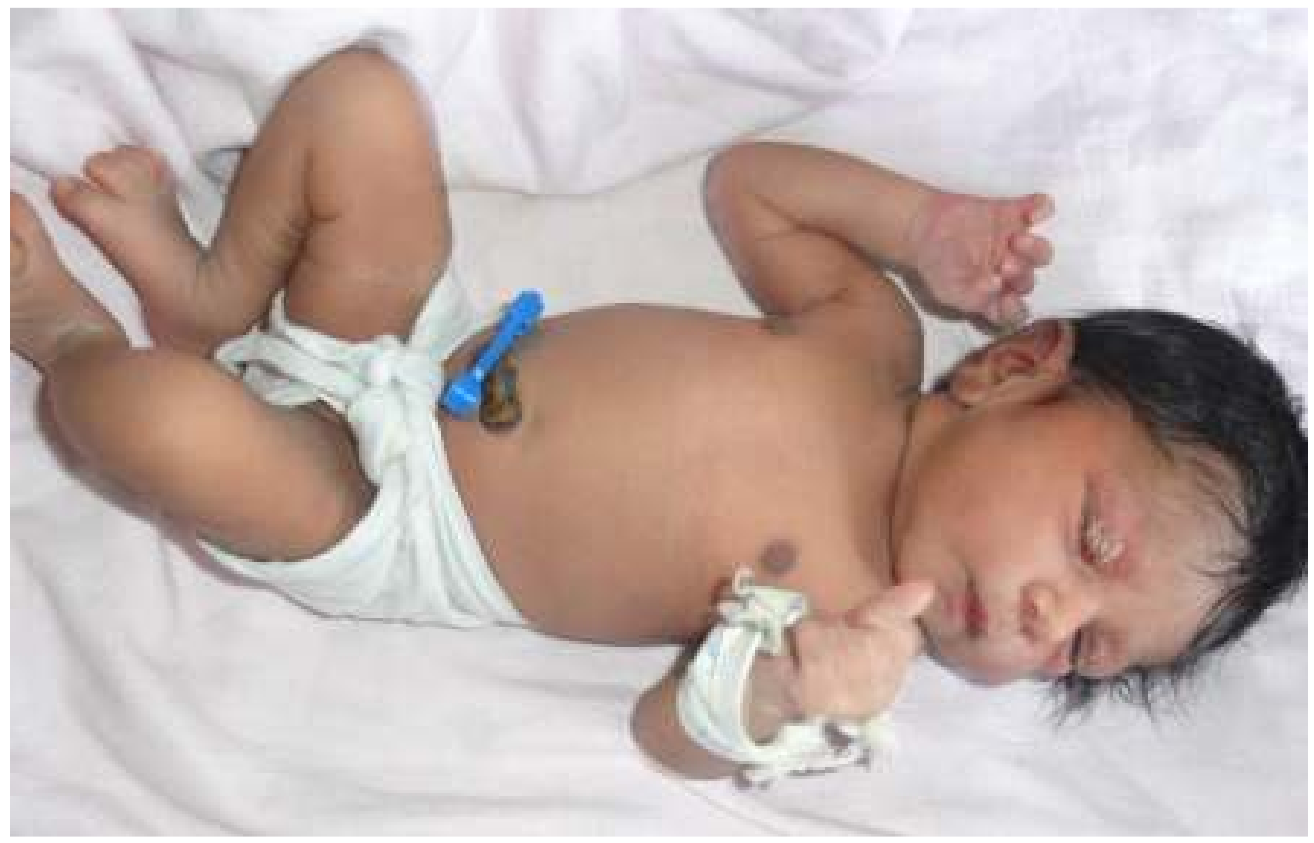

Figure 1: Discrete pustules localised to upper eyelids and pinna

\section{${ }^{1}$ Department of Paediatrics, ${ }^{2}$ Department of Dermatology, Hindu Rao Hospital, India}

(Received on 18 September 2013: Accepted after revision on 25 October 2013)
Giemsa stained smears from pustules were prepared on two separate occasions, from two different sites each time and observed by three different microscopists. All the smears showed eosinophilic predominance $(70 \%)$. Gram's stain and potassium hydroxide preparation of pustules failed to reveal any microorganisms. 
The rash resolved on its own after three days without any residual signs. No sequelae were observed at 1 month.

\section{Discussion}

Typical lesions of ETN are maculo-papular evolving into firm, pale yellow or white pustules 1$3 \mathrm{~mm}$ in diameter surrounded by red and swollen background skin giving a 'flea-bitten' appearance ${ }^{3}$. They appear on the trunk, extremities and face and heal without sequel after 5-7 days. The palms and soles are rarely involved. In our patient, atypical features included well circumscribed distribution, pustular predominance and absence of perilesional erythema in almost all of the lesions. Features consistent with ETN included typical pustularsmears, with a corroborative clinical course.

Transient neonatal pustular melanosis (TNPM) presents with fragile, superficial, vesiculo-pustules with neutrophils or occasional eosinophils, appearing at birth or within the first day of life. It is most commonly seen in term infants of both sexes of African origin ${ }^{9}$. Lesions decompress leaving pigmented macules surrounded by collarette of scales. There is absence of peri-pustular oedema. In our patient, the rash appeared on the first day and there was absence of 'flea-bitten' appearance but eosinophilic predominance and the lack of scales or residual hyperpigmentation contradict this diagnosis.

Eosinophilic pustular folliculitis ${ }^{10}$ presents as recurrent crops of papulo-pustular lesions on the trunk and extremities with predilection for scalp and hair follicles. Pustular and peripheral eosinophilia may be seen. Absence of scalp lesions, scaling, crusting, as well as the lack of recurrence argues against this diagnosis in our patient.

Infantile acropustolosis ${ }^{11}$ presents as recurrent crops of 1-2 mm pruritic vesiculo-pustules, rich in neutrophils and eosinophilis, on the distal extremities between the ages of 2 and 10 months, though onset in the neonatal period has been reported. Characteristic distribution, lack of pruritus as well as non-recurrent nature in our case, makes this diagnosis unlikely.

\section{References}

1. Liu C, Feng J, Qu R, Zhou H, Ma H, Niu X, et al. Epidemiologic study of the predisposing factors in erythema toxicum neonatorum. Dermatology 2005; 210(4):269-72. http://dx.doi.org/10.1159/000084749
2. O'Connor NR, McLaughlin MR, Ham P. Newborn skin: Part I. Common rashes. American Family Physician 2008; 77(1):4752.

3. Schwartz RA, Janniger CK. Erythema toxicum neonatorum. Cutis 1996; 58(2):153-5.

4. Keitel HG, Yadav V. Etiology of toxic erythema. Erythema toxicum neonatorum. American Journal of Diseases of Children 1963; 106:306-9.

http://dx.doi.org/10.1001/archpedi.1963.02080 $\underline{050308010}$

5. Carr JA, Hodgman JE, Freedman RI, Levan NE. Relationship between toxic erythema and infant maturity. American Journal of Diseases of Children 1966; 112(2):129-34.

6. Levy HL, Cothran F. Erythema toxicum neonatorum present at birth. American Journal of Diseases of Children 1962; 103:617-9.

7. Hurwitz S. Clinical pediatric dermatology, 2nd ed. Philadelphia: WB Saunders, 1993:13-14.

8. Bassukas ID. Is erythema toxicum neonatorum a mild self-limited acute cutaneous graftversus-host-reaction from maternal-to-fetal lymphocyte transfer? Medical Hypotheses 1992; 38(4):334-8. http://dx.doi.org/10.1016/03069877(92)90028$\underline{B}$

9. Laude TA. Approach to dermatologic disorders in black children Seminars in Dermatology 1995; 14(1):15-20. http://dx.doi.org/10.1016/S10855629(05)8003 $\underline{4-3}$

10. Ofuji S, Ogino A, Horio T, Oseko T, Uehara M. Eosinophilic pustular folliculitis. Acta Dermato Venereologica 1970; 50(3):195-203.

11. Kahn G, Rywlin AM. Acropustulosis of infancy. Archives of Dermatology 1979; 115 (7):831-3 http://dx.doi.org/10.1001/archderm.115.7.831 